
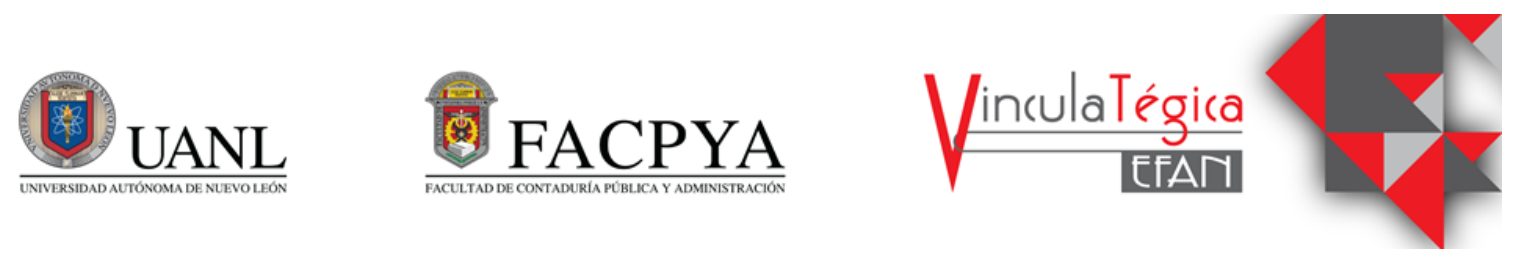

\title{
Operaciones simuladas: EFOS y EDOS (Artículo 69-B del Código Fiscal de la Federación)
}

\author{
Noé Francisco Rico Martínez y Adriana Verónica Hinojosa Cruz ${ }^{2}$ \\ ${ }^{1}$ Universidad Autónoma de Nuevo León, Monterrey, Nuevo León, México, noe.rico77@ hotmail.com, (044) \\ 8180196162. \\ ${ }^{2}$ Universidad Autónoma de Nuevo León, Nuevo León, México, avhinojosacruz@gmail.com, (044) \\ 8182803799.
}

Información del artículo revisado por pares

Fecha de aceptación: junio-2021

Fecha de publicación en línea: diciembre-2021

DOI: https://doi.org/10.29105/vtga7.2-24

\section{Resumen}

La presente ponencia tuvo como objetivo describir el artículo $69-\mathrm{B}$ del CFF, como medida de contrarrestar esquemas de prácticas fiscales indebidas a través de operaciones simuladas que utilizan las empresas EFOS y EDOS. Se utilizó estadística descriptiva y se presenta los resultados recolectados en una tabla y en una gráfica de pastel en porcentaje. La población fueron las empresas que se encuentran en la lista de contribuyentes del SAT, de acuerdo con la reforma donde se adiciona y se aprueba el artículo 69-B del Código Fiscal de la Federación, con un total de 12505 empresas presuntas de operaciones simuladas, de los cuales 10519 son empresas definitivas que representan un $42 \%$, es decir, que no lograron desvirtuar los hechos, así mismo, 903 empresas tienen sentencia favorable que representan un $4 \%$, es decir, que interpusieron un medio de defensa, del mismo modo, 783 empresas están como presuntos, lo que representa un $3 \%$, por lo que tendrán desvirtuar los hechos y por último, 301 empresas desvirtuados, que representan el $1 \%$, ya que manifestaron ante la autoridad fiscal la suficiente información y documentación para
The objective of this presentation was to describe Article 69 - B of the CFF, as a measure to counteract schemes of improper tax practices through simulated operations used by EFOS and EDOS companies. Descriptive statistics were used and the results collected are presented in a table and in a pie graph in percentage. The population was the companies that are on the list of taxpayers of the SAT, in accordance with the reform where article 69-B of the Federal Tax Code is added and approved, with a total of 12,505 companies alleged to have simulated operations, of which 10,519 are definitive companies that represent $42 \%$, that is, they failed to disprove the facts, likewise, 903 companies have a favorable judgment that represent $4 \%$, that is, they filed a defense, in the same way, 783 companies are as alleged, which represents $3 \%$, so they will have to distort the facts and finally, 301 distorted companies, which represent $1 \%$, since they presented enough information and documentation to the tax authority to distort the facts. The results obtained between 2014 and 2018 due to the misuse of digital tax receipts online, derived from non-existent operations in Mexico, identified 8,204 
desvirtuar los hechos.Los resultados obtenidos entre los años 2014 y 2018 por el uso indebido de comprobantes fiscales digitales por internet, derivado de operaciones inexistentes en México, se identificaron a 8204 empresas factureras, las cuales generaron 8 millones de facturas apócrifas, lo cual genero significativamente un monto de $\$ 354$ mil 512 millones de pesos de evasión fiscal y que impactan en la recaudación fiscal y a su vez tiene consecuencias significativas del $1.4 \%$ en el PIB. Sin embargo, en los resultados obtenidos con base al análisis de datos históricos, se concluyó que la lista de contribuyentes del SAT se ha incrementado, es decir, la problemática continua, ya que de los años 2014 a 2018 fueron de 8204 a 12505 empresas al 18 de mayo del 2021.

Palabras clave: EDOS, EFOS, facturación apócrifa, operaciones simuladas.

\section{INTRODUCCIÓN}

Hoy en día, las operaciones simuladas y/o inexistentes son una realidad. Son consideradas como un delito grave para las administraciones tributarias, y tienen efectos significativos en la evasión fiscal.

Por otro lado, el uso indebido de comprobantes fiscales por medio de facturas apócrifas, derivado a través de operaciones simuladas, va en contra de lo que dice la ley y para la autoridad tributaria mexicana se considera una práctica fiscal indebida, que afecta intereses no sólo del fisco federal, sino de la sociedad en general al verse reducida la recaudación de ingresos, pues recordemos que en México en el Artículo 31 fracción IV de la Constitución Política de los Estados Unidos Mexicanos (CPEUM), establece que "es obligación de los mexicanos contribuir a los gastos públicos, así de la Federación, como del Distrito Federal o del Estado y Municipio en que residan, de la manera proporcional y equitativa que dispongan las leyes" (CPEUM, 2020).

En México, en el año 2014 surgen las figuras fiscales de Empresas que Facturan invoicing companies, which generated 8 million apocryphal invoices, which significantly generated an amount of \$ 354 thousand 512 million pesos of tax evasion and that impact on tax collection and in turn have significant consequences of $1.4 \%$ on GDP. However, in the results obtained based on the analysis of historical data, it was concluded that the list of taxpayers of the SAT has increased, that is, the problem continues, since from the years 2014 to 2018 there were from 8,204 to 12,505 companies per year. May 18, 2021.

Keywords: EODS, EFOS, apocryphal billing, simulated operations. JEL: H32, E26, E27, E62, K34

Operaciones Simuladas EFOS y Empresas que Deducen Operaciones Simuladas EDOS, que describen a las empresas que utilizan como medio para el tráfico de comprobantes fiscales digitales por internet ilegales, considerando operaciones simuladas y a la vez obteniendo un beneficio en perjuicio del fisco federal.

Por lo anterior, la definición de EFOS, son las empresas encargadas de generar facturas apócrifas y/o ficticias de venta de productos o servicios con el objetivo de generar gastos a otras empresas para su beneficio, evitando así el pago de contribuciones y por lo cual figuran en el entendido de evasión de impuestos (SAT, 2014).

Asimismo, los EDOS son las encargadas de contabilizar las transacciones generadas por los EFOS para poder registrar gastos inexistentes y de esta forma evitar o disminuir el pago de impuestos a las autoridades fiscales (SAT, 2014).

No obstante, en el Código Fiscal de la Federación (CFF), no señala el concepto de simulación fiscal. Por tal motivo, "A falta de norma fiscal expresa, se aplicarán 
supletoriamente las disposiciones del derecho federal común cuando su aplicación no sea contraria a la naturaleza del derecho fiscal" (CFF, 2020).

Por consiguiente, se aplica supletoriamente el Código Civil para el Distrito Federal (CCDF). El cual se fundamenta en su artículo 2180, como norma jurídica un acto simulado a aquel “...a las partes declaran o confiesan falsamente lo que en realidad no ha pasado o no se ha convenido entre ellas" (CCDF, 2020).

De acuerdo con la exposición de motivos del ejecutivo federal, donde se reforma y adiciona el artículo 69 del Código Fiscal de la Federación (CFF), lo cual fue implementado y aprobado, como una medida de control para contrarrestar los esquemas de evasión fiscal, limitando la posibilidad de incurrir en prácticas indebidas que determinados contribuyentes realizan al considerar la deducción de gastos ficticios amparados a través de un Comprobante Fiscal Digital por Internet (CFDI), permitiéndoles disminuir la base gravable y determinar un impuesto a cargo menor, nulo o en su caso, un saldo a favor al momento de presentar las declaraciones (Gaceta parlamentaria, 2013).

Por consiguiente, la Comisión de Hacienda y Crédito Público, con proyecto de decreto por el que se reforman, adicionan y derogan diversas disposiciones del Código Fiscal de la Federación, publica con fecha de Martes 10 de Septiembre de 2013, los argumentos que sustentan la Iniciativa, de acuerdo a la exposición de motivos del Ejecutivo Federal, respecto del Código Fiscal de la Federación, lo siguiente:

Por lo antes mencionado y bajo ese contexto, la iniciativa propone modificar:

- El artículo 17 - H del Código Fiscal de la Federación (CFF), por medio de medidas contra contribuyentes defraudadores, con el fin de dejar sin efectos los certificados de sellos o firmas digitales emitidos por contribuyentes, por utilizar comprobantes fiscales digitales para amparar probables operaciones inexistentes, simuladas o ilícitas (CFF,
2014).

- Así mismo, el artículo 69 del CFF, a efecto de establecer la facultad de la autoridad fiscal para publicar en su página de internet el nombre, denominación o razón social y la clave del registro federal de contribuyentes de aquellos con quien es riesgoso celebrar actos mercantiles o de comercio, porque no cumplen con sus obligaciones fiscales, con el fin de que tengan elementos para decidir las operaciones que lleven a cabo entre contribuyentes cumplidos e incumplidos (CFF, 2014).

- Del mismo modo, en el citado artículo 69 del CFF, se propone establecer un procedimiento para el uso indebido de comprobantes fiscales, para sancionar y neutralizar las prácticas de los contribuyentes que realizan el tráfico de comprobantes fiscales, específicamente para quienes los adquieran, vendan o colocan y quienes de alguna manera se benefician de este tipo de actividad ilegal que tanto afecta al fisco federal (CFF, 2014).

Cabe mencionar, que no todos los contribuyentes se ubican en el supuesto de emisores o receptores de comprobantes fiscales con operaciones inexistentes $\mathrm{o}$ simuladas; y aun cuando en aquellos casos en los que la autoridad fiscal presuma la inexistencia de determinadas operaciones de un contribuyente, éste tiene el derecho de desvirtuar los hechos que llevaron a la autoridad a encuadrarlo en tal supuesto.

Por lo anterior, el Servicio de Administración Tributaria (SAT) abre una ventana con la finalidad de garantizar los derechos de los contribuyentes, y generarles certeza jurídica en el procedimiento relativo a la presunción de operaciones inexistentes que lleva a cabo la autoridad fiscal, mediante el Decreto publicado en el DOF el 25 de junio de 2018, se reformó y aprobó el artículo 69-B del CFF, iniciando vigencia a los treinta días siguientes a su publicación, estableciéndose, entre otros supuestos, nuevos plazos para desvirtuar los hechos y para emitir la resolución por parte de la 
autoridad, así como la publicación del listado de contribuyentes que obtengan sentencia favorable (Gaceta parlamentaria, 2013).

La presente ponencia tiene como objetivo describir el artículo $69-\mathrm{B}$ del CFF, como medida de contrarrestar esquemas de prácticas fiscales indebidas a través de Operaciones Simuladas que utilizan las empresas EFOS y EDOS.

H1: Con la implementación y aprobación del artículo 69 - B del Código Fiscal de la Federación al que hace referencia a operaciones simuladas, si contrarresta las prácticas indebidas de las empresas EFOS y EDOS en México, durante el periodo de tiempo 2014 a 2023.

\section{MARCO TEÓRICO}

En este apartado se definirán los conceptos que contextualizan la presente ponencia.

\subsection{Derechos y principios tributarios}

"La contribución es un concepto genérico que engloba a todo lo que auxilia a sufragar los gastos del Estado" (Rodríguez, 2020).

El Derecho Tributario se define como "a la rama de Derecho Público que estudia los principios, fija las normas que rigen la creación y percepción de los tributos y determina la naturaleza y los caracteres de las relaciones del Estado con el administrado que está obligado a pagarlos" (Andreozzi, 2020).

Principio de Proporcionalidad y Equidad o Justicia "Los súbditos de cada Estado deben contribuir al sostenimiento del gobierno en una proporción lo más cercana posible a sus respectivas capacidades: es decir, en proporción a los ingresos de que gozan bajo la protección del Estado. De la observancia o menosprecio de esa máxima depende lo que se llama la equidad o falta de equidad de los impuestos" (Smith, 2020).

$\mathrm{Al}$ respecto, Zavala (2020) señala que "los impuestos se deben establecer por medio de leyes, tanto desde el punto de vista material, como del formal, es decir, por medio de disposiciones de carácter general, abstractas, impersonales y emanadas del
Poder Legislativo".

\subsection{Evasión fiscal}

Para Folco (2005) define la evasión fiscal como "todo acto u omisión que, en contradicción con la ley fiscal aplicable al mismo, provoca indebidamente la disminución o eliminación de la denominada carga tributaria".

Por su parte Flores (2004) "la evasión fiscal es el incumplimiento de un deber legal que surge del hecho generador del crédito fiscal, es decir es un ilícito que lleva aparejadas consecuencias de carácter legal, y se puede clasificar en dos formas: la legal y la ilegal, es el conjunto de actos o artificios realizados por un contribuyente para eludir el cumplimiento de disposiciones tributarias a su cargo".

Según Macon (1987) "el crecimiento de la evasión generalizada trae consigo el aumento de las tasas para compensarlo, con lo cual la diferencia entre los que pagan y los que no lo hacen se acentúa, es decir que no solamente la evasión es un proceso autoalimentado por actos de imitación, sino también fomentado por la política fiscal que reacciona en la única forma que puede".

Según González (2002) "La evasión de impuestos surge debido a una decisión del contribuyente de no declarar, total o parcialmente, determinadas operaciones o ingresos que debería acumular para los respectivos efectos fiscales. La evasión fiscal se vuelve injusta por el hecho de que sólo una parte de la población paga por la provisión de los servicios públicos que todos disfrutan y, por lo tanto, conlleva una carga efectiva mucho más elevada para quienes si cumplen con sus obligaciones fiscales".

"Evasión fiscal o tributaria es toda eliminación o disminución de un monto tributario producido dentro del ámbito de un país por parte de quienes están jurídicamente obligados a abonarlo y que logran tal resultado mediante conductas fraudulentas u omisivas violatorias de disposiciones legales" (Villegas, 1993). 


\subsection{Empresas que Facturan Operaciones Simuladas EFOS y Empresas que Deducen Operaciones Simuladas EDOS}

EFOS son las empresas encargadas de generar facturas apócrifas y/o ficticias de venta de productos o servicios con el objetivo de poder generar gastos a otras empresas para su beneficio, evitando así el pago de impuestos y por lo cual figuran en el entendido de evasión de impuestos (SAT, 2020).

Asimismo, los EDOS son las encargadas de contabilizar las transacciones generadas por los EFOS para poder registrar gastos inexistentes y de esta forma evitar o disminuir el pago de impuestos a las autoridades fiscales (SAT, 2020).

No obstante, estás empresas están constituidas conforme a la Ley General de Sociedades Mercantiles (LGSM), es decir, se constituyen ante notario público, están inscritos en el Registro Público de la Propiedad y en el Registro Federal de Contribuyentes (RFC), tienen domicilio fiscal establecido, cuentan con firma electrónica avanzada y expiden comprobantes fiscales digitales por internet a través de la plataforma del SAT. Incluso, algunas de estas empresas pagan cantidades menores de impuestos para ocultar sus actos delictivos (Ureste, Castillo, \& Roldan, 2018, pág. 170).

\subsection{Factura electrónica}

Una factura apócrifa o una simulación de operaciones son, en primera instancia, un documento falso, es decir, una factura en la que de manera intencional se ha puesto información incorrecta. Esta información incorrecta podría referirse a: la naturaleza de la transacción que ocurrió; el monto de lo facturado; los detalles de la compañía o el agente emisor, y los detalles del consumidor o receptor (IOTA, 2009)

Gómez (1998, p. 55), en su obra La simulación de los Negocios jurídicos define a la simulación como "la declaración o un contenido de voluntad no real emitida conscientemente y de acuerdo entre las partes para producir, con fines de engaño, la apariencia de un negocio jurídico que no existe o es distinto de aquel que realmente se ha llevado a cabo".

Para (López, Espinoza, \& Acosta, 2012), la Factura electrónica es "un mecanismo de comprobación fiscal que se basa en el aprovechamiento de medios electrónicos para la generación, procesamiento, transmisión y resguardo de los documentos fiscales de manera digital".

Según García (2014), la factura constituye una de las herramientas de información con las que cuenta la administración tributaria, para ejercer un control efectivo el cumplimiento de los deberes tributarios, además de que la factura se configura como requisito para aplicar determinados gastos y deducciones y para el ejercicio de los derechos de repercusión y deducción del impuesto sobre el valor agregado (IVA).

Para Banda (2016), una factura es un documento de tipo mercantil que se utiliza para compilar la información relacionada respecto a la compra y la venta de un producto o servicio. Este concepto involucra detalles precisos acerca de la operación en cuestión, de este modo una factura, sirve para demostrar y justificar la entrega de un producto o servicio después de su compra. El tipo y modelo de factura cambia dependiendo del producto o servicio del que se trate, de la forma de compra y de las necesidades de cada momento.

Según Rasteletti (2018), en el SAT se han implementado 3 modelos de facturación históricamente:

- El esquema en papel con la intervención de un tercero inicia en la década de los 90 a 2013.

- El esquema de la factura electrónica, 2004-2011

- El esquema de la factura electrónica por Internet, 2011-2017

\section{MÉTODO}

En este apartado se describirá el método que fundamenta la validez de la presente ponencia, se define el enfoque y alcance de la misma

Se inició la investigación literaria 
consultando fuentes de información por internet para encontrar artículos relacionados con el tema de la ponencia utilizando las palabras claves "edos", "efos", "facturación apócrifa" y "operaciones simuladas".

A continuación, en la indagación por internet se utilizaron las comillas "", con el fin de encontrar artículos con las disertaciones exactas y así reducir los resultados de la búsqueda.

Por otro lado, se consultaron de igual manera documentos oficiales, artículos de investigación y estudios de proyectos realizados sobre operaciones simuladas.

\section{RESULTADOS}

Este apartado se analiza la información obtenida de acuerdo al listado de contribuyentes conforme a la reforma donde se adiciona y aprueba el artículo $69-\mathrm{B}$ del CFF.

El Servicio de Administración Tributaria, ha sostenido que se obtuvo una afectación por 354 mil millones de pesos, derivado de la operación de empresas que facturan operaciones simuladas (SAT, 2020).

Asimismo, según el SAT, en la simulación de operaciones en México entre 2014 al 2018 se identificaron a ocho mil 204 EFOS, las cuales generaron más de ocho millones de facturas falsas por un monto de 1.6 billones de pesos que se traducen en la evasión fiscal de 354 mil 512 millones de pesos, cifra que asciende al 1.4 del Producto Interno Bruto (SAT, 2021).

Tabla 1. Operaciones simuladas en México periodo 2014 a 2018.

\begin{tabular}{|c|c|c|c|c|}
\hline Años & $\begin{array}{c}\text { Empresas } \\
\text { Efos }\end{array}$ & $\begin{array}{c}\text { Generación } \\
\text { de facturas } \\
\text { falsas }\end{array}$ & $\begin{array}{c}\text { Monto de } \\
\text { evasión } \\
\text { fiscal }\end{array}$ & PIB \\
\hline $\begin{array}{c}2014 \\
-\end{array}$ & 8 mil 204 & 8 millones & $\begin{array}{c}354 \text { mil } \\
512 \\
\text { millones } \\
\text { de pesos }\end{array}$ & $1.4 \%$ \\
\hline
\end{tabular}

Fuente: Elaboración propia con datos del SAT

Sin embargo, el SAT, el listado de contribuyentes conforme a la reforma donde se adiciona y aprueba el artículo 69 - B del CFF, al día 18 de mayo de 2021, se tuvo un incremento con un total de 12505 empresas presuntas de la inexistencia de operaciones simuladas (SAT, 2021).

Por lo anterior, se muestra la siguiente gráfica:

Gráfica 1. Listado de contribuyentes de empresas presuntas de operaciones simuladas del periodo 2014 a 2021.

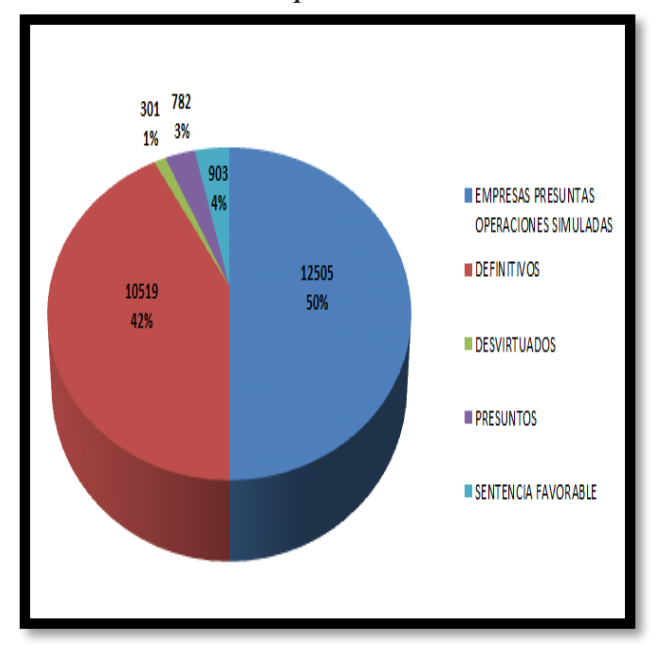

Fuente: Elaboración propia con datos del SAT

Se observó un total 12505 empresas presuntas de la inexistencia de operaciones simuladas que representan el 50\% de la lista de contribuyentes del SAT, de los cuales 10519 son empresas definitivas que representan un $42 \%$, es decir, que no lograron desvirtuar los hechos, así mismo, 903 empresas tienen sentencia favorable que representan un $4 \%$, es decir, que interpusieron un medio de defensa, del mismo modo, 783 empresas están como presuntos, lo que representa un $3 \%$, por lo que tendrán desvirtuar los hechos y por último, 301 empresas desvirtuados, que representan el $1 \%$, ya que manifestaron ante la autoridad fiscal la suficiente información y documentación para desvirtuar los hechos.

\section{CONCLUSIONES}

Los resultados obtenidos con base al análisis de datos históricos, reflejan que aun y cuando la autoridad hace mención a aquellos contribuyentes defraudadores de cancelar los certificados de sellos o firmas digitales, publicar en la lista de 
contribuyentes del SAT, el nombre, razón social y la clave del registro federal de contribuyentes de aquellos con quien es riesgoso celebrar actos de comercio, la problemática continua, sigue existiendo y se ha incrementado la lista de contribuyentes de los años 2014 a 2018 de 8204 a 12505 empresas al 18 de mayo del 2021.

Pese a los esfuerzos realizados por promover la iniciativa de reforma y la aprobación del artículo 69 - B del CFF, la problemática continua y es que siguen existiendo las mismas estrategias que algunos contribuyentes usan para evadir el pago de impuestos a través, por ejemplo, la facturación apócrifa, que sigue haciendo estragos en la recaudación, como medio de erosión de la base imponible.

Asimismo, con la implementación a la normatividad de este procedimiento se pretendió desde un inicio combatir dicha problemática.

Sin embargo, esta medida ha resultado insuficiente para disuadir y acabar con esta práctica ilegal y nociva para el fortalecimiento de las finanzas públicas. (Vázquez, 2019).

Por lo anterior, con la implementación y aprobación del artículo 69 - B del Código Fiscal de la Federación al que hace referencia a operaciones simuladas, no contrarresta las prácticas indebidas de las empresas EFOS y EDOS en México, durante el periodo de tiempo 2014 a 2023.

Sin duda alguna, para el gobierno federal, las operaciones simuladas EFOS y EDOS, significan un gran reto en las administraciones actuales y siguientes, ya que es un problema que afecta prácticamente a la mayoría de las economías del mundo, en diferente medida y motivada por diversas circunstancias, su presencia tiene consecuencias negativas sobre el crecimiento y desarrollo de los países.

\subsection{PROPUESTAS}

Se proponen acciones para implementar la reducción de comprobantes fiscales que amparan operaciones simuladas y/o inexistentes:

- Implementar estrategias de fiscalización para combatir el tráfico de comprobantes fiscales digitales.

- Fortalecimiento de la Ley para abordar temas, como el de la facturación apócrifa EFOS y EDOS.

- Capacitar y adiestrar a la autoridad federal para el fortalecer y mejorar la eficiencia recaudatoria, así como implementar acciones de verificación en cuanto a empresas que cuenten con el recursos humano, financiero y material, para poder operar el negocio, inclusive la toma de fotografías para verificar el domicilio fiscal establecido que manifiesta el contribuyente.

- Incrementar la tasa fija de impuesto corporativo a empresas en general que hayan incurrido en algún delito en operaciones simuladas y/o inexistentes a través de empresas factureras EFOS y EDOS. 


\section{REFERENCIAS}

Andreozzi Manuel "Derecho tributario Argentino" editorial tipográfica argentina, 1951

Cámara de Diputados del H. Congreso de la Unión. Dirección General de Bibliotecas. Código Fiscal de la Federación. [en línea]. México, D.F. Última Reforma DOF 30-01-2007. [Fecha de consulta: 02 febrero 2007]. Disponible en: http://www.diputados.gob.mx/leyinfo/pdf/8.pdf

Código Civil de Comercio del Distrito Federal. Recuperado de: https://leyes$\mathrm{mx} . c 0 \mathrm{~m} /$ codigo_civil_federal/2180.htm

Código Fiscal de la Federación, publicado en el Diario Oficial de la Federación. Recuperado de: http://gaceta.diputados.gob.mx/

Constitución Política de los Estados Unidos mexicanos. Recuperado de: https://www.scjn.gob.mx/sites/default/files/transparencia/documentos/becarios/188jorgeluis-revilla-de-la-torre.pdf

Derecho fiscal segunda edición Raúl Rodríguez Lobato UNIVERSIDAD NACIONAL AUTÓNOMA DE MÉXICO

Flores Zavala, Ernesto. "Elementos de Finanzas Públicas Mexicanas”, 13ª ed. Porrúa, México, 1971

Folco, Carlos María, "El fenómeno de la evasión fiscal”, en Calos María Folco, Sandro F. Abraldes y Javier López Biscayart, Ilícitos Fiscales. Asociación ilícita en materia tributaria, Rubinzal - Culzoni Editores, Buenos Aires, 2004, p. 17.

García, A. M. (2014). La regulación de la factura electrónica en el sector público. Redalyc.org, 12.

Gómez (1998). “La simulación de los negocios jurídicos”. Pag. 55

González, F (2002) Derecho Fiscal Mexicano, pag. 242 en ARRIOJA VIZCAINO, A., Derecho Fiscal, Ed. Themis, México, Sep.1998, p.488.

IOTA. (2009). False and fictitious Invoices: Report for tax asministrations

López, E., Espinoza, E., \& Acosta, M. (2012). Tecnología de la información: Impacto de la Factura electrónica. CISCI, 6.

Macon, J (1987). Medida de la evasión, economía no registrada. En: Publicaciones del INDEC, Buenos Aires Argentina, julio.

Rasteletti, A (2018) Perfil de la Factura Electrónica. En A. Barreix \& Zambrano (Eds.), Factura Electrónica en América Latina, (pp. 83-90). doi; http//dx.doi.org/10.18235/0001038

Servicio de Administración tributaria. Recuperado el día 28 de Mayo de 2021 de: http://omawww.sat.gob.mx/cifras_sat/Paginas/datos/vinculo.html?page=ListCompleta69B.h tml

Smith A (1961) “La riqueza de las naciones” Enciclopedia Británica, edición 1911

Vázquez Caro, J (1993). "Como influir en la conciencia tributaria del contribuyente para mejorar su comportamiento". Trabajo presentado ante la Asamblea General del CIAT, Chile

Villegas, H., Arguello Velez, G., \& Spila Jarcia, R. (1993). La evasión fiscal en Argentina. Revista de Derecho Fiscal No XXIII - Pág 337. 